\title{
Individual variation in susceptibility or exposure to SARS-CoV-2 lowers the herd immunity threshold
}

\author{
Authors: M. Gabriela M. Gomes ${ }^{1 *}$, Ricardo Aguas ${ }^{2}$, Jessica G. King ${ }^{3}$, Kate E. Langwig ${ }^{4}$, \\ Caetano Souto-Maior ${ }^{5}$, Jorge Carneiro ${ }^{6}$, Carlos Penha-Gonçalves ${ }^{6}$, Guilherme Gonçalves ${ }^{7}$, \\ Marcelo U. Ferreira ${ }^{8}$.
}

${ }^{1}$ Department of Mathematics and Statistics, University of Strathclyde, Glasgow, United Kingdom.

${ }^{2}$ Centre for Tropical Medicine and Global Health, Nuffield Department of Medicine, University of Oxford, Oxford, United Kingdom.

${ }^{3}$ Institute of Evolutionary Biology, University of Edinburgh, Edinburgh, United Kingdom.

${ }^{4}$ Department of Biological Sciences, Virginia Tech, Blacksburg, VA, USA.

${ }^{5}$ Laboratory of Systems Genetics, National Heart Lung and Blood Institute, National Institutes of Health, Bethesda, MD, USA.

${ }^{6}$ Instituto Gulbenkian de Ciência, Oeiras, Portugal.

$15{ }^{7}$ Unidade Multidisciplinar de Investigação Biomédica, Instituto de Ciências Biomédicas Abel Salazar, Universidade do Porto, Porto, Portugal.

${ }^{8}$ Instituto de Ciências Biomédicas, Universidade de São Paulo, São Paulo, Brazil.

*Correspondence to: gabriela.gomes@strath.ac.uk.

Abstract: Severe acute respiratory syndrome coronavirus 2 (SARS-CoV-2) began to spread in late 2019, gradually depleting susceptible populations and causing the incidence of new infections to decline over time. Variation in individual susceptibility or exposure to infection exacerbated this effect. Individuals who were more susceptible or more exposed tended to be infected earlier, depleting the susceptible subpopulation of those who were at higher risk of infection. This selective depletion of susceptibles intensified the deceleration in incidence irrespectively of vaccination programmes. In a theoretical scenario of acquired immunity being long-lasting, susceptible numbers would eventually become low enough to prevent epidemic growth or, in other words, the herd immunity threshold (HIT) would be naturally reached. Early in the coronavirus disease (COVID-19) pandemic, simple calculations, relying on homogeneity assumptions, suggested that herd immunity would require $60-80 \%$ of the population to become immune. Here we build, into the underlying models, individual variation in susceptibility or in exposure to infection and demonstrate how that lowers the HIT. Based on data from a variety of sources and early parameter estimates these basic models suggest the HIT associated with natural infection to be in the order of $10-30 \%$ for SARS-CoV-2. We discuss how a structure accounting for unobserved selectable variation can be a core feature of infectious disease modelling embedded in model development as reality evolves and complexity is incremented. The goal is to enhance accuracy and enable trade-offs to be adequately addressed in the design of optimal policies. 
medRxiv preprint doi: https://doi.org/10.1101/2020.04.27.20081893; this version posted August 1, 2021. The copyright holder for this preprint (which was not certified by peer review) is the author/funder, who has granted medRxiv a license to display the preprint in perpetuity.

It is made available under a CC-BY-NC-ND 4.0 International license .

\section{Introduction}

Severe acute respiratory syndrome coronavirus 2 (SARS-CoV-2) emerged in China in late 2019 and spread worldwide causing the ongoing pandemic of coronavirus disease (COVID-19). Scientists throughout the world have engaged with governments, health agencies, and with each other, to address this emergency. Mathematical models have been central to important decisions concerning contact tracing, quarantine, and social distancing, to mitigate or suppress the initial pandemic spread (1). Successful suppression, however, leaves populations at risk to resurgent waves due to insufficient acquisition of immunity by natural infection. Early models have thus also addressed longer term SARS-CoV-2 transmission scenarios and the requirements for continued adequate response (2). During the first year of the pandemic the world was also stage to intense vaccine research, with several vaccines becoming available in record time. Countries began to vaccinate their populations in late 2020 / early 2021.

Here we address a specific weakness shared by mathematical models used early in the pandemic and discuss consequences downstream. Models such as $(1,2)$ assume that all individuals in a population are identical. While this simplification is sensible for most traits, homogenizing susceptibility and exposure to infection causes models to overpredict pandemic potential and overestimate the impact of various interventions that may be enacted. In the interest of clarity our analysis is focused on the simplest early models developed prior to vaccination or viral evolution concerns. Our results are nevertheless fundamental leading to conclusions that are preserved as these, and other complexities are added to the models.

We demonstrate that individual variation in susceptibility or exposure (connectivity) accelerates the acquisition of natural immunity in populations due to selection by the force of infection. More susceptible and more connected individuals have a higher propensity to be infected and thus are likely to become immune earlier. Due to this selective immunization, heterogeneous populations require less infections to cross their herd immunity threshold (HIT) than homogeneous (or not sufficiently heterogeneous) models would suggest. To illustrate these ideas, we integrate continuous distributions of susceptibility or connectivity in otherwise basic epidemic models for COVID-19 and show that as the coefficient of variation (CV) describing the level of population heterogeneity in either susceptibility or connectivity increases from 0 to 4 , the herd immunity threshold declines from over $60 \%(3,4)$ to less than $10 \%$. Given that empirical estimates of CV for SARS-CoV-1 and SARS-CoV-2 span this range, the identified sensitivity highlights the importance of seeking relevant measures of individual variation to inform transmission models.

The direct measurement of individual variation in susceptibility is not straightforward and to our knowledge it has not been conducted for SARS-CoV-2. Individual variation in exposure is to some extent measurable through contact investigations (5). In a recent analysis ( 6 ), based on 20 contact surveys conducted throughout the world, CV has been assessed to be near 1 (mean 0.9, standard deviation 0.2) and gamma distributions have been shown to provide good descriptions of that variation.

\section{SARS-CoV-2 transmission in heterogeneous populations}

SARS-CoV-2 is transmitted primarily by respiratory droplets and modelled as a susceptibleexposed-infectious-recovered (SEIR) process. 
medRxiv preprint doi: https://doi.org/10.1101/2020.04.27.20081893; this version posted August 1, 2021. The copyright holder for this preprint (which was not certified by peer review) is the author/funder, who has granted medRxiv a license to display the preprint in perpetuity.

Individual variation in susceptibility is integrated as a continuously distributed factor that multiplies the force of infection upon individuals as

$$
\dot{S}(x)=-\lambda x S(x), \quad \dot{E}(x)=\lambda x S(x)-\delta E(x), \quad \dot{I}(x)=\delta E(x)-\gamma I(x),
$$

where $S(x)$ is the number of individuals with susceptibility $x, E(x)$ and $I(x)$ are the numbers of individuals who originally had susceptibility $x$ and became exposed and infectious, $\delta$ is the rate of progression from exposed to infectious, $\gamma$ is the rate of recovery or death, and $\lambda=$ $(\beta / N) \int[\rho E(x)+I(x)] d x$ is the average force of infection upon susceptible individuals in a population of size $N . \rho$ denotes the infectiousness of individuals in $E(x)$ in relation to those in $I(x)$, and $\beta$ is a transmission coefficient defined as the mean number of contacts per person per time unit. The basic reproduction number is

$$
R_{0}=\langle x\rangle \beta(\rho / \delta+1 / \gamma)
$$

where $\langle x\rangle$ is the mean susceptibility factor at epidemic onset. Prior to the epidemic, susceptibility is described by a probability density function $q(x)$ with mean $\langle x\rangle=1$ and $C V=\sqrt{\left\langle(x-1)^{2}\right\rangle}$ explored as a parameter.

In a directly transmitted infectious disease, such as COVID-19, variation in exposure to infection is primarily governed by patterns of connectivity among individuals. We incorporate this in the system (Equation 1) by modifying the force of infection as $\lambda=$ $(\beta / N)\left(\int x[\rho E(x)+I(x)] d x / \int x q(x) d x\right)$ and the basic reproduction number as

$$
R_{0}=\left(\left\langle x^{2}\right\rangle /\langle x\rangle\right) \beta(\rho / \delta+1 / \gamma)
$$

where $\langle x\rangle=1$ and $\left\langle x^{2}\right\rangle$ are the first and second moments of the distribution $q(x)$ prior to the epidemic.

Like other modelling practiced early in the pandemic (1) these basic structures do not consider reinfection. They nevertheless enable the derivation of conclusions that remain valid when waning immunity enabling reinfection is implemented $(6,7)$.

\section{The herd immunity threshold}

Individual variation in risk of acquiring infection is under selection by the force of infection, whether differences are due to inherent biological susceptibility, physical exposure, or a combination of the two. Selection results in the removal of the most at-risk individuals from the susceptible pool as they become infected and eventually recover with immunity (or die). This selective acquisition of infection and immunity results simultaneously in decelerated epidemic growth and accelerated induction of immunity in the population.

The herd immunity threshold (HIT) defines the percentage of the population that needs to be immune (due to natural infection, in this construction) to reverse epidemic growth and prevent future waves of infection. When individual susceptibility or connectivity is gamma distributed, HIT can be derived as a function of $R_{0}$ and $\mathrm{CV}$ as in (7). In the case of variation in susceptibility we obtain

$$
H I T=1-\left(\frac{1}{R_{0}}\right)^{\frac{1}{1+C V^{2}}}
$$

while variable connectivity results in 


$$
H I T=1-\left(\frac{1}{R_{0}}\right)^{\frac{1}{1+2 C V^{2}}} .
$$

Figure 1 shows the expected downward trends in the HIT for SARS-CoV-2 as the coefficients of variation of the gamma distribution are increased between 0 and 4 . To assess sensitivity to the type of distribution we show equivalent plots with lognormal distributions in Figure 2. The latter were calculated numerically as the percentage of the population no longer susceptible when the rate of change in new infections $(\dot{E})$ crosses from positive to negative. While HIT requires 60 $80 \%$ of a homogeneous population to be immune given an $R_{0}$ between 2.5 and 5 , these percentages drop sharply with increasing $\mathrm{CV}$, especially when variation is in connectivity. Therefore, it is critically important to know the human profiles of susceptibility and exposure to SARS-CoV-2.

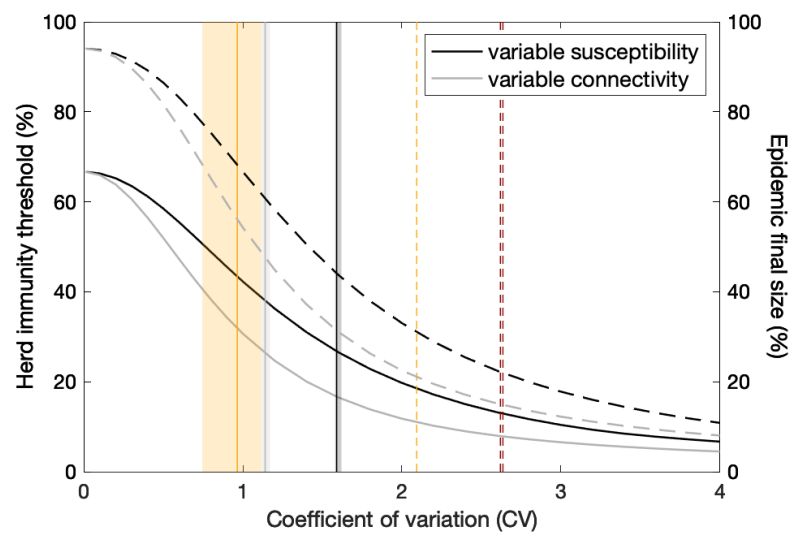

Figure 1: Herd immunity threshold with gamma distributed susceptibility and exposure to infection. Curves generated using the model (Equation 1) with gamma distributed susceptibility (black) or connectivity (grey) assuming $R_{0}=3$ : (solid) herd immunity threshold calculated according to (Equation 4) and (Equation 5); (dashed) final size of unmitigated epidemic. Other parameters: $\gamma=\delta=0.25$ per day $(8-11) ; \rho=0.5$ (12-15). Vertical lines indicate coefficients of individual variation obtained from the literature: (red) infectiousness for SARS-CoV-1 [Singapore 2.62, Beijing 2.64 (16)]; (dashed orange) infectiousness for SARS-CoV-2 [Hong Kong 2.09 (17)] (assuming $R_{0}=3$ ); (solid orange) connectivity [mean 0.93, standard deviation 0.19] (6); (black) susceptibility for SARS-CoV-2 (England and Scotland 1.59) (6); (grey) connectivity for SARS-CoV-2 (England and Scotland 1.14) (6).

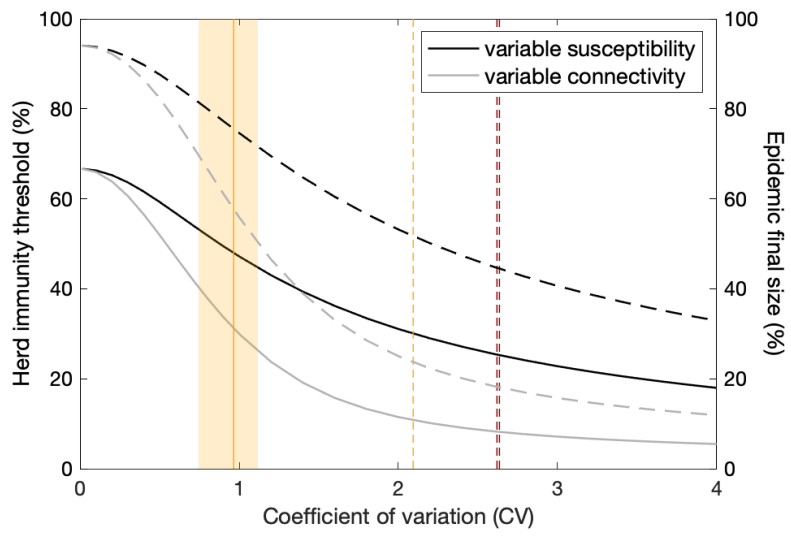


medRxiv preprint doi: https://doi.org/10.1101/2020.04.27.20081893; this version posted August 1, 2021. The copyright holder for this preprint (which was not certified by peer review) is the author/funder, who has granted medRxiv a license to display the preprint in perpetuity. It is made available under a CC-BY-NC-ND 4.0 International license .

Figure 2: Herd immunity threshold with lognormal distributed susceptibility and exposure to infection. Curves generated with the model (Equation 1) with lognormal distributed susceptibility (black) or connectivity (grey) assuming $R_{0}=3$ : (solid) herd immunity threshold calculated as the percentage of the population no longer susceptible when the rate of change $\dot{E}$ crosses from positive to negative; (dashed) final size of unmitigated epidemic. Other parameters: $\gamma=\delta=0.25$ per day $(8-11) ; \rho=0.5$ (12-15). Vertical lines indicate coefficients of individual variation obtained from the literature: (red) infectiousness for SARS-CoV-1 [Singapore 2.62, Beijing 2.64 (16)]; (dashed orange) infectiousness for SARS-CoV-2 [Hong Kong 2.09 (17)] (assuming $R_{0}=3$ ); (solid orange) connectivity [mean 0.93 , standard deviation 0.19 ] (6).

We searched the literature and found no empirical estimates of individual variation in susceptibility to coronaviruses but identified studies measuring variation in the number of secondary SARS-CoV-1 or SARS-CoV-2 infections attributed to single infected individuals $(16,17)$. CV estimates from these studies (2.6 for SARS-CoV-1, and 2.1 for SARS-CoV-2) are overlaid as vertical dashed lines in Figures 1 and 2. This type of variation has been implied in the occurrence of scarce and explosive outbreaks due to superspreading $(16,17)$, but it remains unclear how responsive it is to the kind of selection that lowers the HIT. Variation in infectiousness does not respond to selection while variation in connectivity can respond, especially when propensity for social contacts is a persistent characteristic of individuals. Typical superspreading investigations are not designed to characterise the relative contributions of biological infectiousness and physical connectivity. Hence, we must assume that both contribute to the measured variation and interpret results from these studies as overestimates of the selectable connectivity variation. Another kind of study, which measures contact patterns directly (5), enables such decomposition, in principle. Not surprisingly, such studies provide lower CV estimates (0.9), as marked by the solid orange line. These are likely to be underestimations, however, since they do not include individual variation in susceptibility whose measurement is nigh impossible. Comparing the coloured lines with the heterogeneous connectivity HIT curve in the figures (grey) we note that all intersections occur when HIT is roughly in the range $10-30 \%$.

Limitations of direct individual-based studies, for our purposes, have prompted us to develop indirect procedures to infer selectable variation from specific population-based designs $(18,19)$. In the case of SARS-CoV-2 the inferences were conducted by fitting a model (Equation 1) to daily series of COVID-19 deaths in England and Scotland (from the beginning of the pandemic until 1 February 2021, capturing first and second pandemic waves), resulting in CV estimates between those obtained by contact surveys and those reported by superspreading studies ( 6 ). These were performed with gamma-distributed susceptibility and resulted in $C V=1.59$ with the variable susceptibility formulation (black) and $C V=1.14$ when variation was formulated in connectivity (grey). A combined formulation should result in estimates within this range. Once sufficient selection has occurred in the pandemic, these inferences become robust over different time scales. The analysis was replicated on shorter series (until 1 July 2020, to capture the first pandemic wave only) leading to similar estimates. This contrasts with results from Tkachenko et al. (20) on the effects of time scales of individual variation on COVID-19 dynamics.

\section{Discussion}

The concept of herd immunity is commonly used in the design of vaccination programs $(21,22)$. Defining the percentage of the population that must be immune to cause infection incidences to decline, herd immunity thresholds constitute convenient targets for vaccination coverage. In idealized scenarios of vaccines delivered at random and individuals mixing at random, herd 
medRxiv preprint doi: https://doi.org/10.1101/2020.04.27.20081893; this version posted August 1, 2021. The copyright holder for this preprint (which was not certified by peer review) is the author/funder, who has granted medRxiv a license to display the preprint in perpetuity. It is made available under a CC-BY-NC-ND 4.0 International license .

immunity thresholds are given by a simple formula $\left(1-1 / R_{0}\right)$ which, in the case of SARSCoV-2, suggests that $60-80 \%$ of the population would need to be immunized to halt spread considering early estimates of $R_{0}$ between 2.5 and 5. A crucial caveat in exporting these calculations to immunization by natural infection is that natural infection does not occur at random. Individuals who are more susceptible or more exposed are more prone to be infected and become immune, which lowers the threshold $(23,24)$. In the absence of vaccination, HIT declines sharply when coefficients of variation in susceptibility and/or connectivity in our model increase from 0 to 2 and remains low for more variable populations. The amplitude of the decline depends on the exact source of heterogeneity and how it is distributed but the downward trend is robust.

Heterogeneity in the transmission of respiratory infections has traditionally focused on variation in exposure summarized into age-structured contact matrices $(5,25)$. Besides overlooking differences in susceptibility given exposure, the aggregation of individuals into age groups reduces coefficients of variation which may have important downstream implications (6). It is therefore crucial that variation in susceptibility and exposure to infection is included in epidemic models at the finest resolution. Individual variation has traditionally been addressed resorting to agent-based models which are computationally intensive (26). Here, we use mathematical formalisms that enable the entire individual variation to be captured while maintaining the analytical tractability of the simplest homogeneous models.

Public health measures and behavioural changes can themselves alter individual variation. Social distancing may be argued to either increase or decrease variation in exposure, depending on the roles of different functional groups in societies and the compliance of individuals who are normally more highly connected in relation to the average. Datasets that describe connectivity patterns before and during movement restrictions, such as those in (27) could, in principle, inform relevant changes in distributions of individual connectivity but surveys must be applied on representative samples of the population and the information should be collapsed into agegroup averages. Although a deeper understanding of the putative patterns would be valuable for refining control strategies and interpreting data resulting from prevalence studies and serological surveys, this does not appear essential to the development of predictive epidemic models providing selectable variation can be inferred from epidemic dynamics.

We have assumed acquired immunity to be long-lasting in the analyses presented here as others have done early in the pandemic ( 1$)$. There is nevertheless evidence that significant reinfection has been occurring with SARS-CoV-2 (25), which we have addressed elsewhere $(6,7)$. The derivation of HIT formulas in (7) adopts a general formalism that includes reinfection while, in (6), COVID-19 death series in England and Scotland were fitted using models with and without reinfection. When reinfection is moderately enabled, all parameters readjust to fit the data and projections of future trajectories remain close to those obtained without reinfection.

More than one year through the pandemic, detailed analyses of realistic scenarios require models with waning immunity, viral evolution, seasonality, vaccination, spatial structure, etc. The heterogeneity structures described here can be built into any such model to enhance the accuracy that enables trade-offs to be adequately addressed in the design of optimal policies. Herd immunity thresholds can be rederived for any such model and associated epidemic trajectories can be projected under specific scenarios. 
medRxiv preprint doi: https://doi.org/10.1101/2020.04.27.20081893; this version posted August 1, 2021. The copyright holder for this preprint (which was not certified by peer review) is the author/funder, who has granted medRxiv a license to display the preprint in perpetuity. It is made available under a CC-BY-NC-ND 4.0 International license .

To be able to generate reliable predictions, a model must account for the effects of selection on individual variation, but this does not require fully mechanistic implementations. In general, the amount of variation that responds to selection by the force of infection can be inferred from the shape of epidemic curves and used to forecast future trajectories under plausible scenarios.

\section{References:}

1. S. Flaxmax, S. Mishra, A. Gandy, H. J. T. Unwin, T. A. Mellan, H. Coupland, et al., Estimating the effects of non-pharmaceutical interventions on COVID-19 in Europe. Nature 584, 257-261 (2020).

2. S. M. Kissler, C. Tedijanto, E. Goldstein, Y. H. Grad, M. Lipsitch, Projecting the transmission dynamics of SARS-CoV-2 through the postpandemic period. Science 368, 860868 (2020).

3. J. T. Wu, K. Leung, G. M. Leung, Nowcasting are forecasting the potential domestic and international spread of the 2019-nCov outbreak originating in Wuhan, China: a modelling study. Lancet 395, 689-697 (2020).

4. K. O. Kwok, F. Lai, W. I. Wei, S. Y. S. Wong, J. Tang, Herd immunity - estimating the level required to halt the COVID-19 epidemics in affected countries. J. Infect. 80, e32-e33 (2020).

5. J. Mossong, N. Hens, M. Jit, P. Beutels, K. Auranen, R. Mikolajczyk, et al., Social contacts and mixing patterns relevant to the spread of infectious diseases. PLOS Med. 5, e74 (2008).

6. M. G. M. Gomes, M. U. Ferreira, M. Chikina, W. Pegden, R. Aguas, Frailty variation models for susceptibility and exposure to SARS-CoV-2. medRxiv: 10.1101/2021.05.25.21257766 (2021).

7. A. Montalbán, R. M. Corder, M. G. M. Gomes, Herd immunity under individual variation and reinfection. arXiv 200800098v2. (2020).

8. S. A. Lauer, K. H. Grantz, Q. Bi, F. K. Jones, Q. Zheng, H. R. Meredith, et al., The Incubation Period of Coronavirus Disease 2019 (COVID-19) From Publicly Reported Confirmed Cases: Estimation and Application. Ann. Intern. Med. 172, 577-582 (2020).

9. Q. Li, X. Guan, P. Wu, X. Wang, L. Zhou, Y. Tong, et al., Early transmission dynamics in Wuhan, China, of novel coronavirus-infected pneumonia. N. Engl. J. Med. 382, 1199-1207 (2020).

10. J. Zhang, M. Litvinova, W. Wang, Y. Wang, X. Deng, X. Chen, et al., Evolving epidemiology and transmission dynamics of coronavirus disease 2019 outside Hubei province, China: A descriptive and modelling study. Lancet Infect. Dis. 20, 793-802 (2020).

11. H. Nishiura, N. M. Linton, A. R. Akhmetzhanov, Serial interval of novel coronavirus (COVID-19) infections. Int. J. Infect. Dis. 93, 284-6 (2020).

12. W. E. Wei, Z. Li, C. J. Chiew, S. E. Yong, M. P. Toh, V. J. Lee, Presymptomatic Transmission of SARS-CoV-2 - Singapore, January 23-March 16, 2020. MMWR Morb. Mortal. Wkly. Rep. 69, 411-415 (2020).

13. K. K. W. To, O. T. Y. Tsang, W. S. Leung, A. R. Tam, T. C. Wu, D. C. Lung, et al., Temporal profiles of viral load in posterior oropharyngeal saliva samples and serum antibody 
medRxiv preprint doi: https://doi.org/10.1101/2020.04.27.20081893; this version posted August 1, 2021. The copyright holder for this preprint (which was not certified by peer review) is the author/funder, who has granted medRxiv a license to display the preprint in perpetuity. It is made available under a CC-BY-NC-ND 4.0 International license .

responses during infection by SARS-CoV-2: an observational cohort study. Lancet Infect. Dis. 20, 565-74 (2020).

14. M. M. Arons, K. M. Hatfield, S. C. Reddy, A. Kimball, A. James, J. R. Jacobs, et al., Presymptomatic SARS-CoV-2 infections and transmission in a skilled nursing facility. $N$. Engl. J. Med. 382, 2081-2090 (2020).

15. X. He, E. H. Y. Lau, P. Wu, X. Deng, J. Wang, X. Hao, et al., Temporal dynamics in viral shedding and transmissibility of COVID-19. Nat. Med. 26, 672-675 (2020).

16. J. O. Lloyd-Smith, S. J. Schreiber, P. E. Kopp, W. M. Getz, Superspreading and the effect of individual variation on disease emergence. Nature 438, 355-359 (2005).

17. D. C. Adam, P. Wu, J. Y. Wong, E. H. Y. Lau, T. K. Tsang, S. Cauchemez, et al., Clustering and superspreading potential of SARS-CoV-2 infections in Hong Kong. Nat. Med. 26, 17141719 (2020).

18. M. G. M. Gomes, M. Lipsitch, A. R. Wargo, G. Kurath, C. Rebelo, G. F. Medley, et al., A missing dimension in measures of vaccination impacts. PLOS Pathog. 10, e1003849 (2014).

19. M. G. M. Gomes, A pragmatic approach to account for individual risks to optimise health policy. arXiv:2009.01354 (2020)

20. A. V. Tkachenko, S. Maslov, A. Elbanna, G. N. Wong, Z. J. Weiner, G. Goldenfeld, Timedependent heterogeneity leads to transient suppression of the COVID-19 epidemic, not herd immunity. Proc. Natl. Acad. Sci. U. S. A. 118, e2015972118 (2021).

21. G. Gonçalves, Herd immunity: recent uses in vaccine assessment. Expert Rev. Vaccines 7, 1493-1506 (2008).

22. P. Fine, K. Eames, D. L. Heymann, "Herd immunity": a rough guide, Clin. Infect. Dis. 52, 911-916 (2011).

23. M. J. Ferrari, S. Bansal, L. A. Meyers, O. N. Bjornstad, Network frailty and the geometry of herd immunity. Proc. R. Soc. B 273, 2743-2748 (2006).

24. T. Britton, F. Ball, P. Trapman, A mathematical model reveals the influence of population heterogeneity on herd immunity to SARS-CoV-2. Science 369, 846-849 (2020).

25. K. Prem. A. R. Cook, M. Jit, Projecting social contact matrices in 152 countries using contact surveys and demographic data. PLOS Comput. Biol. 13, e1005697 (2017).

26. S. Eubank, H. Guclu, V. S. A. Kumar, M. V. Marathe, A. Srinivasen, Z. Toroczkai, et al., Modelling disease outbreaks in realistic urban social networks. Nature 429, 180-184 (2004).

27. J. Zhang, M. Litvinova, Y. Liang, Y. Wang, S. Zhao, Q. Wu, et al., Changes in contact patterns shape the dynamics of the COVID-19 outbreak in China. Science 10.1126/science.abb8001 (2020).

28. V. J. Hall, S. Foulkes, A. Charlett, A. Atti, E. J. Monk, R. Simmons, et al., SARS-CoV-2 infection rates of antibody-positive compared with antibody-negative health-care workers in England: a large, multicentre, prospective cohort study (SIREN). Lancet 397, 1459-1469 (2021).

Acknowledgements: We thank Rodrigo Corder and Antonio Montalbán for contributions to 
medRxiv preprint doi: https://doi.org/10.1101/2020.04.27.20081893; this version posted August 1, 2021. The copyright holder for this preprint (which was not certified by peer review) is the author/funder, who has granted medRxiv a license to display the preprint in perpetuity. It is made available under a CC-BY-NC-ND 4.0 International license .

Funding: MUF received funding from Conselho Nacional de Desenvolvimento Científico e Tecnológio (CNPq), Brazil. CSM was supported by the Intramural Research Program of the $\mathrm{NIH}$, The National Heart Lung and Blood Institute.

Author contributions: MGMG conceived the study and wrote the first draft, MGMG and JGK performed the analyses, all authors wrote the paper.

Competing interests: Authors declare no competing interests. 